\title{
Temperature and pH Stability Profiles of ortho and para DEET
}

\author{
Mirine Choi, Seok Yong Kim, Joonho Choi ${ }^{1}$, Sang Hee Park, \\ Chul Soon Yong ${ }^{2}$ and Jung Sun Kim ${ }^{\dagger}$ \\ Department of Biotechnology \& Division of Health Science, Dongseo University, Busan 617-716, South Korea \\ ${ }^{1}$ College of Pharmacy, Seoul National University, Seoul, Korea \\ ${ }^{2}$ College of Pharmacy, Yeungnam University, 214-1 Dae-Dong, Gyongsan 712-749, South Korea \\ (Received May 23, $2011 \cdot$ Revised June 10, $2011 \cdot$ Accepted August 11, 2011)
}

\begin{abstract}
DEET, $N, N$ '-diethyl- $m$-toluamide, is the most commonly used mosquito repellent. However, it can easily permeate through skin leading to toxic effects. A recent study showed that the ortho analogue of DEET showed enhanced repellency with reduced permeation compared to the commercially used meta analogue. Thus, in order to understand the differences in properties and effectiveness among the $m$-, $o$ - and $p$-analogues of DEET, an HPLC-UV method was developed for separately analyzing the three analogues. Moreover, stability profiles at temperatures ranging from $30^{\circ} \mathrm{C}$ to $70^{\circ} \mathrm{C}$ as well as $\mathrm{pH}$ ranging from $\mathrm{pH} 3$ to $\mathrm{pH} 9$ have been determined. All three analogues were stable with no degradation observed during the 5 day period. $o$-DEET therefore could be further developed into a safer and more effective mosquito repellent.
\end{abstract}

Key words - DEET, Temperature stability, pH stability, Mosquito repellency

$m$-DEET is known as the golden standard of mosquito repellents for being highly effective, stable and relatively safe (Katz et al., 2008). In most cases it is directly applied to the human skin protecting against tick-borne diseases (Jensennius et al., 2005) and mosquito-mediated diseases including dengue fever, West Nile virus and Eastern Equine Encephalitis (Gubler, 2001). However, information about the mechanism of action of DEET is still controversial. Studies report that DEET may block behavior of mosquitoes by inhibiting chemo-receptors on the mosquito antennae that are stimulated by lactic acid (Dogan et al., 1999) whereas other studies refute this phenomenon (Syed and Leal, 2008). Nevertheless, $m$-DEET exhibits mosquito repellency as shown in the complete protection time (CPT) ranging from 203 to 756 minutes, varying with factors including climatic effects, mosquito species and physical activities (Barnard and Xue, 2004; Bidlingmayer, 1994; Bernier et al., 2000).

The search for safer and more effective repellents continues to progress, however, due to the toxicities reported of DEET (Schofield et al., 2007). Possible substitutes to DEET that have been reported so far include synthetically prepared Picaridin, IR3535 and Permethrin or lemon eucalyptus or citronella which are natural products (Katz et al., 2008). Unfortunately, none of these alternatives seem to exhibit properties that could

\footnotetext{
${ }^{\dagger}$ Corresponding Author :

Tel : +82-51-320-1798, E-mail : jsk@gdsu.dongseo.ac.kr

DOI : 10.4333/KPS.2011.41.4.233
}

be more advantageous to the current DEET products.

Current research for developing repellents make use of systematic bioassays or computer based tools which include a QSAR study of $N$-acyl-piperidines (Katritzky et al., 2008) and a genetic trial of odorant-binding protein manipulation ( $\mathrm{Li}$ et al., 2008). One of the shortcomings of the mosquito repellent investigation is the use of human subjects or living vertebrates in the bioassays (Dogan et al., 1999). However, recently, an efficient in vitro bioassay system has been established where blood substitute and collagen membrane were used instead of human blood and skin (Jahn et. al., 2010). The artificial blood substitute was a slight modification of that used by Kogan for an in vitro feeding assay for maintenance of mosquito colonies (Corazza et al., 2005). Unlike Kogan who utilized stretched parafilm as a skin substitute, Jahn made use of a collagen type membrane (Ditzen et al., 2008) since the parafilm alone could not mimic the physiological skin sufficiently. Moreover, in Jahn's study, skin permeation profiles of DEET and its analogues were examined in correlation to the mosquito repellency, expressed as CPT. Among the DEET analogues tested, compared to the commercially used meta DEET analogue, ortho DEET revealed enhanced repellency with reduced skin permeation rate, implying that toxicity due to skin penetration would be lessened (Jahn et al., 2010).

To estimate the applicability of the ortho DEET analogue, a separation method for the three DEET isomers needed to be established. Because of the similar physicochemical characteristics of the three isomers as revealed in the capacity factors 
determined by HPLC, being able to separately analyze the ortho isomer from the meta isomer has been a challenge (Jahn et al., 2010). In addition, the physical stability of the ortho analogue needs to be established in order to develop the appropriate formulation.

Therefore, in continuation of our search for a safer and more effective mosquito repellent, we herein report on the $\mathrm{pH}$ and temperature stability of ortho-DEET, together with an improved HPLC analysis method.

\section{Materials and Methods}

\section{Chemicals and reagents}

meta-DEET was purchased as DEET(97\%) from Aldrich Chemical Co.(St. Louis, Mo.,USA). The ortho and para isomers were synthesized as reported by Jahn et al. (2010). The structures are shown in Figure 1. Double-distilled water was acquired using a Millipore system. HPLC-grade methanol, water and acetonitrile were purchased from J. T. Baker, USA. Sodium acetate and potassium phosphate for the buffer preparation were obtained from Sigma-Aldrich (St. Louis, Mo., USA) and were of analytical grade or better. Phosphoric acid (85\%, KANTO chemicals) and potassium hydroxide $(85 \%$, TEDIA, Fairfield, OH, USA) were used for the $\mathrm{pH}$ adjustment of the phosphate buffer while sodium hydroxide and glacial acetic acid (Yakuri Pure Chemicals Co., Ltd., Kyoto, Japan) were used for the acetate buffer. All other reagents were purchased from Sigma-Aldrich (St. Louis, Mo., USA) and were of synthetic grade or better.

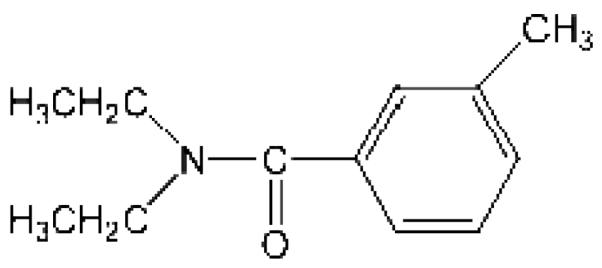

(a)

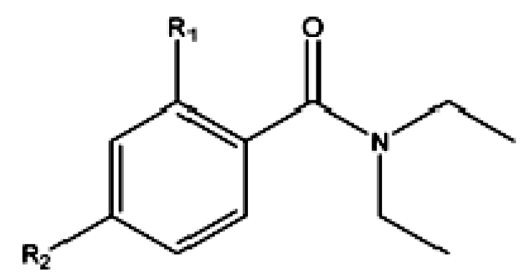

(b)

Figure 1. Chemical Structures of DEET analogues: (a) structure of meta-DEET which is the analogue used in commercial products; (b) ortho DEET where $\mathrm{R}_{1}=\mathrm{CH}_{3}, \mathrm{R}_{2}=\mathrm{H}$ and para DEET where $\mathrm{R}_{1}=\mathrm{H}, \mathrm{R}_{2}=\mathrm{CH}_{3}$.

\section{HPLC analysis}

The liquid chromatographic system consisted of SHIMADZU Solvent Delivery Module LC-10AD (VP) Separation Module and a SHIMADZU UV-Vis Detector SPD-10A(VP) lambda absorbance detector. RP-18 Lichro-cart ${ }^{\circledR} 125$ Lichrosphere $100(5 \mu \mathrm{m}, 150 \times 4 \mathrm{~mm})$ was purchased from MERCK (Darmstadt, Germany) while the $\mathrm{C} 18$ reversed phase column (U6120, S-5 $\mu \mathrm{m}, 4.6 \mathrm{~mm}$ I.D. $250 \times 5 \mathrm{~mm}$ ) was purchased from SHISEIDO (Japan). A SHISEIDO Capcell guard column was also used. The mobile phase consisted of $0.5 \mathrm{M}$ acetate buffers or $0.3 \mathrm{M}$ phosphate buffers of varying $\mathrm{pHs}$ and methanol or acetonitrile and water at varying constitutions. They were filtered through RC membrane filters of $47 \mathrm{~mm}, 0.45 \mu \mathrm{m}$ size (Sartorius, Goettingen, Germany) and degassed in a Branson 1210 Heat Sonic Bath (Model 1210R-DTH). The Corning $\mathrm{pH}$ meter 440 was used for $\mathrm{pH}$ measurements of buffer solutions.

\section{Separation of DEET isomers by HPLC}

Separation of the three analogues of DEET was pursued by finding optimum HPLC conditions by varying the length, inner diameter and packaging of the columns, altering the mobile phase compositions and flow rates. A combination of methanol, water and/or acetonitrile was tested for the mobile phase while the flow rate was varied from 0.6 to $0.9 \mathrm{ml} / \mathrm{min}$. The HPLC condition is as described above with detection at 220 $\mathrm{nm}$ based on a wavelength scan analysis by UV (data not shown). The injection volume was $25 \mu \mathrm{L}$. Resolution factors were calculated using the formula:

$$
R=\left(t_{R 2}-t_{R 1}\right) /\left(w_{1}+w_{2}\right)
$$

where $t_{R}$ is the retention time and $w$ the peak width.

\section{Sample preparation for stability tests}

For $\mathrm{pH}$ stability tests of the meta-, ortho- and para- DEET analogues, buffers of $\mathrm{pH} 3,5,7$, and 9 were made. The buffers of $\mathrm{pH} 3$ and 5 were maintained using a $0.5 \mathrm{M}$ acetate buffer and those of 7 and 9 were maintained using a $0.3 \mathrm{M}$ phosphate buffer, respectively.

All samples were prepared in glass ampoules of which the final volume was $10 \mathrm{~mL}$. Adequate concentrations of DEETs were dissolved in methanol after which the solvent was blown away with nitrogen and filled up to the appropriate volume using buffers of different pHs. For temperature stability studies, the Sanyo drying over (maintained at $70^{\circ} \mathrm{C}$ ) and Hanbaek incubator ( $\mathrm{HB} 201 \mathrm{SF}$, maintained at $30^{\circ} \mathrm{C}$ and $50^{\circ} \mathrm{C}$ ) were used. 
Stability studies were conducted for 5 days. During this 5 day period, samples were removed daily and stored in a $-20^{\circ} \mathrm{C}$ freezer prior to HPLC analysis. At a wavelength of $220 \mathrm{~nm}$, a mobile phase composed of methanol and water $(60: 40, \mathrm{v} / \mathrm{v})$ was used at a flow rate of $0.8 \mathrm{ml} / \mathrm{min}$. RP-18 Lichro-cart ${ }^{\circledR} 125$ LiChrosopher $100(5 \mu \mathrm{m}, 150 \times 4 \mathrm{~mm}$, Merck, Darmstadt, Germany) was used as the stationary phase.

\section{Statistical analysis}

Statistical analysis was performed using a Student t-test at the $\mathrm{p}<0.05$ level. All experiments in the study were repeated at least three times and all data were presented as the mean \pm standard deviation.

\section{In vitro rat skin permeation and deposition study}

The experimental protocols involving animal study were approved by the Animal Care and Use Committee of the College of Pharmacy, Seoul National University. The permission number of animal experiment is "SNU-200909-33." The rat used for the preparation of skin (Sprague Dawley, male, 220$250 \mathrm{~g}$ ) were obtained from OrientBio Co. (Seongnam, Korea). The animals had free access to food and water until used for experiments, and were sacrificed in a $\mathrm{CO}_{2}$ chamber right before the experiments. The dorsal hairs were removed with a clipper and full-thickness skin (about $10 \mathrm{~cm}^{2}$ ) was surgically removed from each rat. The skin specimen was cut into appropriate sizes after carefully removing subcutaneous fat and washing with normal saline. The in vitro skin permeation across the rat skin was conducted with Keshary-Chien diffusion cells at $37^{\circ} \mathrm{C}$. Freshly excised rat skin was mounted between the donor and receptor cell (stratum corneum side facing the donor). The area of diffusion for all in vitro experiments was $2.01 \mathrm{~cm}^{2}$. The receptor cells, which faced the dermis side, were filled with $\mathrm{pH}$ 7.4, $10 \mathrm{mM}$ phosphate buffer solution $(12 \mathrm{~mL})$. At predetermined time intervals, $1 \mathrm{ml}$ of the receptor solution was withdrawn and refilled with the same volume of fresh receptor solution. Samples were kept in a freezer $\left(-20^{\circ} \mathrm{C}\right)$ until analyzed by HPLC. For the skin deposition study, the skins were thoroughly washed with methanol, after which the surface was dried with a cotton swab and DEET content in the dermis skin layer was determined. The effective surface area was separated and minced with a surgical sterile scalpel then finally homogenized with methanol by using ultra turrax homogenizer at $16,000 \mathrm{rpm}$ for $5 \mathrm{~min}$ (T25 Basic, Germany) on ice bath $\left(4^{\circ} \mathrm{C}\right)$. The tissue suspension was centrifuged for $5 \mathrm{~min}$ at $3,000 \times \mathrm{g}$, and then the supernatants were filtered and assayed by HPLC.

\section{Results and Discussion}

\section{Separation of DEET analogues}

The optimum condition for separating the three DEET analogues was best achieved using a reversed phase $\mathrm{C} 18$ column (U6120, S-5 m, $4.6 \mu \mathrm{m}$, ID., $250 \times 5 \mathrm{~mm}$ ) from SHISEIDO (data not shown). However, retention times at which the DEET analogues eluted were $34.34 \mathrm{~min}, 45.24 \mathrm{~min}$ and $46.78 \mathrm{~min}$ for the ortho, meta, and para analogues respectively, which was too long a run for repeated analyses. Thus to decrease the run time, a shorter C18 column of $125 \mathrm{~mm}$ was used even though dispersion occurred to a certain degree. Figure 2 shows the chromatograms where different mobile phase compositions
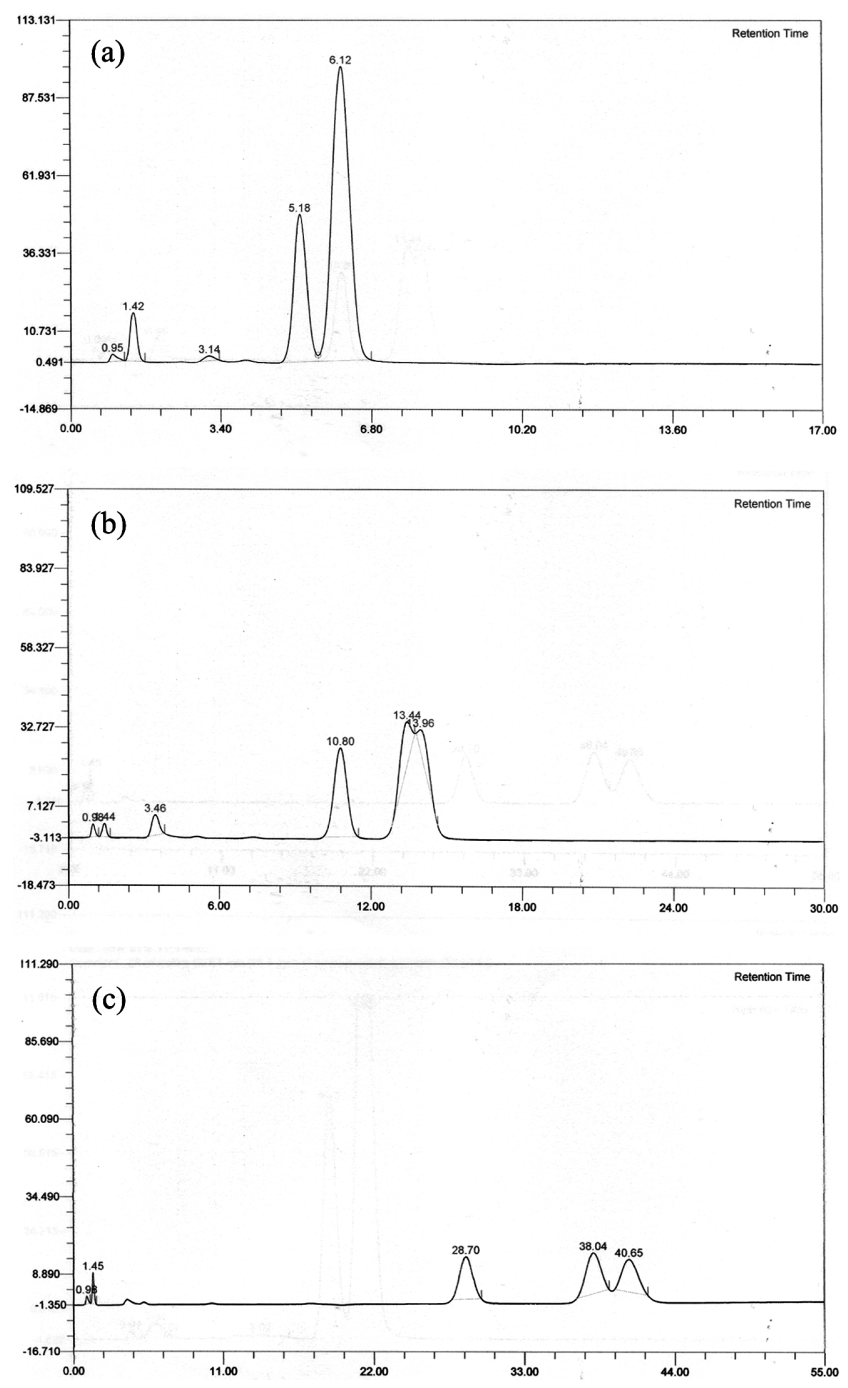

Figure 2. The influence of mobile phase composition on the separation of DEET analogues: (a) Methanol: water (6:4), (b) methanol: water (1:1), (c) methanol: water (4:6). All experiments were done using HPLC-UV $(220 \mathrm{~nm})$ system using RP-18 Lichrocart $^{\circledR} 125$ LiChrosopher 100 at a flow rate of $0.8 \mathrm{ml} / \mathrm{min}$. 
were used when analyses were conducted using this $125 \mathrm{~mm}$ column. The DEET isomers at all times eluted in the order of ortho-, meta- and para- analogues respectively. Moreover, the ortho-DEET at all times did not overlap with the other two peaks whereas resolution of the meta and para was always a challenge. The optimum separation condition was further ascertained by the stipulation and comparison of the resolution factors as shown in Table I. Resolution factors are calculated based on the difference in retention times divided by the sum of the widths of the two peaks as shown in the equation in the Materials and Methods section. The larger the value, the better separation was achieved. A resolution factor of 1.0 was achieved when using methanol: water $(42: 58, \mathrm{v} / \mathrm{v})$ as mobile phase at a flow rate of $0.9 \mathrm{~mL} / \mathrm{min}$. However, satisfactory resolution $(1.0 \pm 0.3)$ was observed with methanol: water (42:58, $\mathrm{v} / \mathrm{v}$ ) at a flow rate of $0.8 \mathrm{~mL} / \mathrm{min}$. Figure 3 shows the rela-

Table I. Resolution factors $(R)$ between meta and para DEET using different mobile phase compositions and varying flow rates with a $250 \mathrm{~mm}$ C18 column

\begin{tabular}{cccc}
\hline \hline Mobile phase & Flow rate & $\mathrm{R}$ & $\begin{array}{c}\text { rt } p \text {-DEET } \\
{[\mathrm{min}]}\end{array}$ \\
\hline MeOH:water [50:50, v/v] & $0.6 \mathrm{~mL} / \mathrm{min}$ & 0.90 & 44.12 \\
& $0.7 \mathrm{~mL} / \mathrm{min}$ & 0.77 & 31.87 \\
& $0.8 \mathrm{~mL} / \mathrm{min}$ & 0.76 & 27.69 \\
MeOH:water [48:52, v/v] & $0.6 \mathrm{~mL} / \mathrm{min}$ & 0.87 & 38.94 \\
& $0.7 \mathrm{~mL} / \mathrm{min}$ & 0.84 & 33.32 \\
& $0.8 \mathrm{~mL} / \mathrm{min}$ & 0.76 & 29.25 \\
MeOH:water [46:54, v/v] & $0.7 \mathrm{~mL} / \mathrm{min}$ & 0.85 & 38.93 \\
& $0.8 \mathrm{~mL} / \mathrm{min}$ & 0.81 & 34.01 \\
MeOH:water [44:56, v/v] & $0.8 \mathrm{~mL} / \mathrm{min}$ & 0.99 & 42.51 \\
& $0.9 \mathrm{~mL} / \mathrm{min}$ & 0.89 & 37.83 \\
MeOH:water [42:58, v/v] & $0.8 \mathrm{~mL} / \mathrm{min}$ & 1.03 & 52.54 \\
& $0.9 \mathrm{~mL} / \mathrm{min}$ & 1.00 & 45.78 \\
\hline
\end{tabular}

rt: retention time

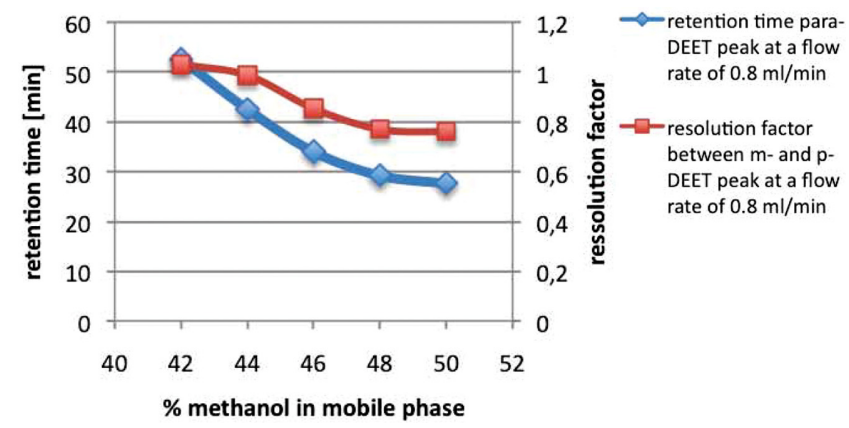

Figure 3. Resolution factor vs. polarity of mobile phase. As the percentage of methanol increased in the mobile phase, better resolution was observed although retention time was delayed. tionship between resolution factor and polarity of the mobile phase (in \% methanol). Although resolution was improved as the polarity of the mobile phase increased, the delay in retention time should be taken into consideration as well especially when routine repeated analysis should be conducted. Therefore, it was decided that for repeated analyses, conditions with lower resolution be utilized after which characterization of the analogue be made using the method where resolution is higher.

\section{Stability tests}

The stability profiles revealed ortho- and para- DEET to be as stable as the commercially used meta-DEET. All three isomers were tested for temperature stability at 30,50 , and $70^{\circ} \mathrm{C}$ as well as for $\mathrm{pH}$ stability at $\mathrm{pHs} 3,5,7$ and 9. As shown in Figures 4 and 5, all three DEET isomers were stable with no significant degradation over 5 days. These results imply the applicability of the DEET isomers as mosquito repellents.
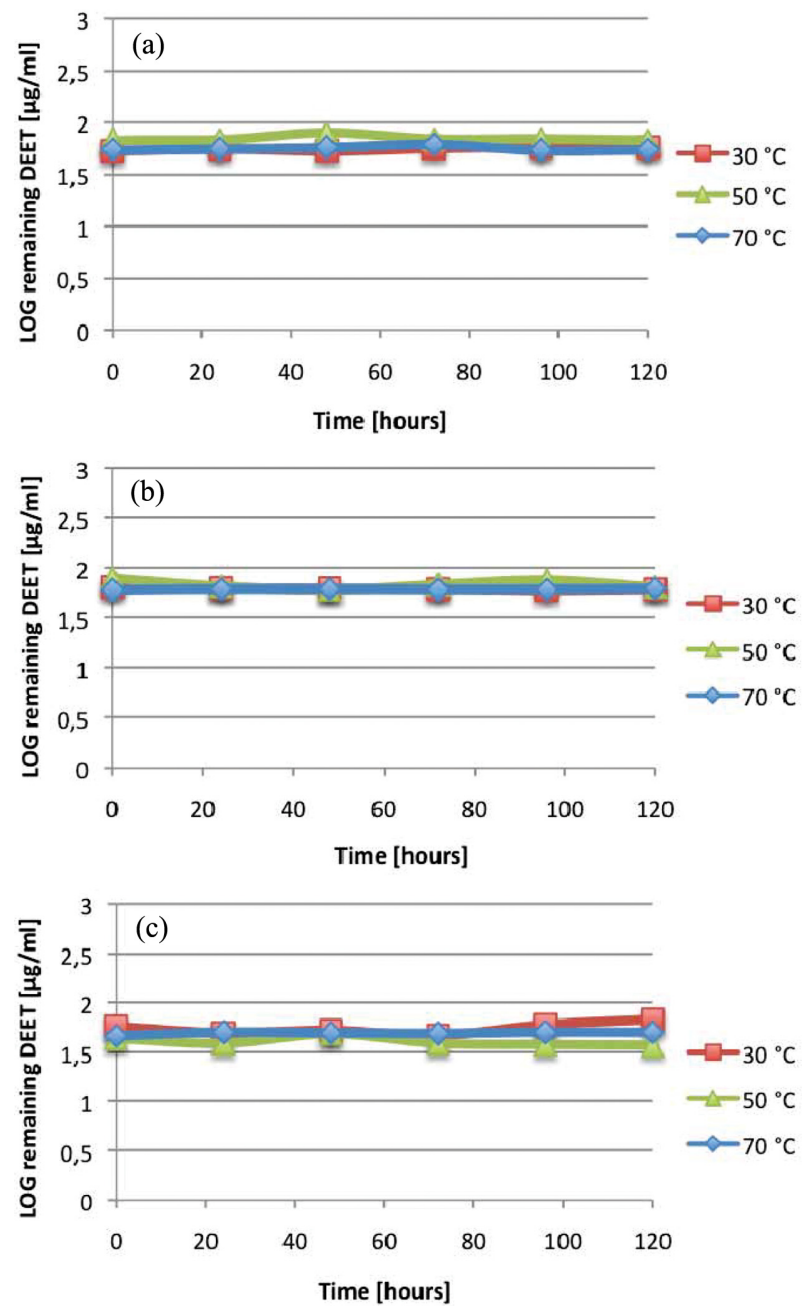

Figure 4. Temperature stability of (a) ortho DEET, (b) para DEET and (c) meta DEET at 30,50 , and $70^{\circ} \mathrm{C}$. 

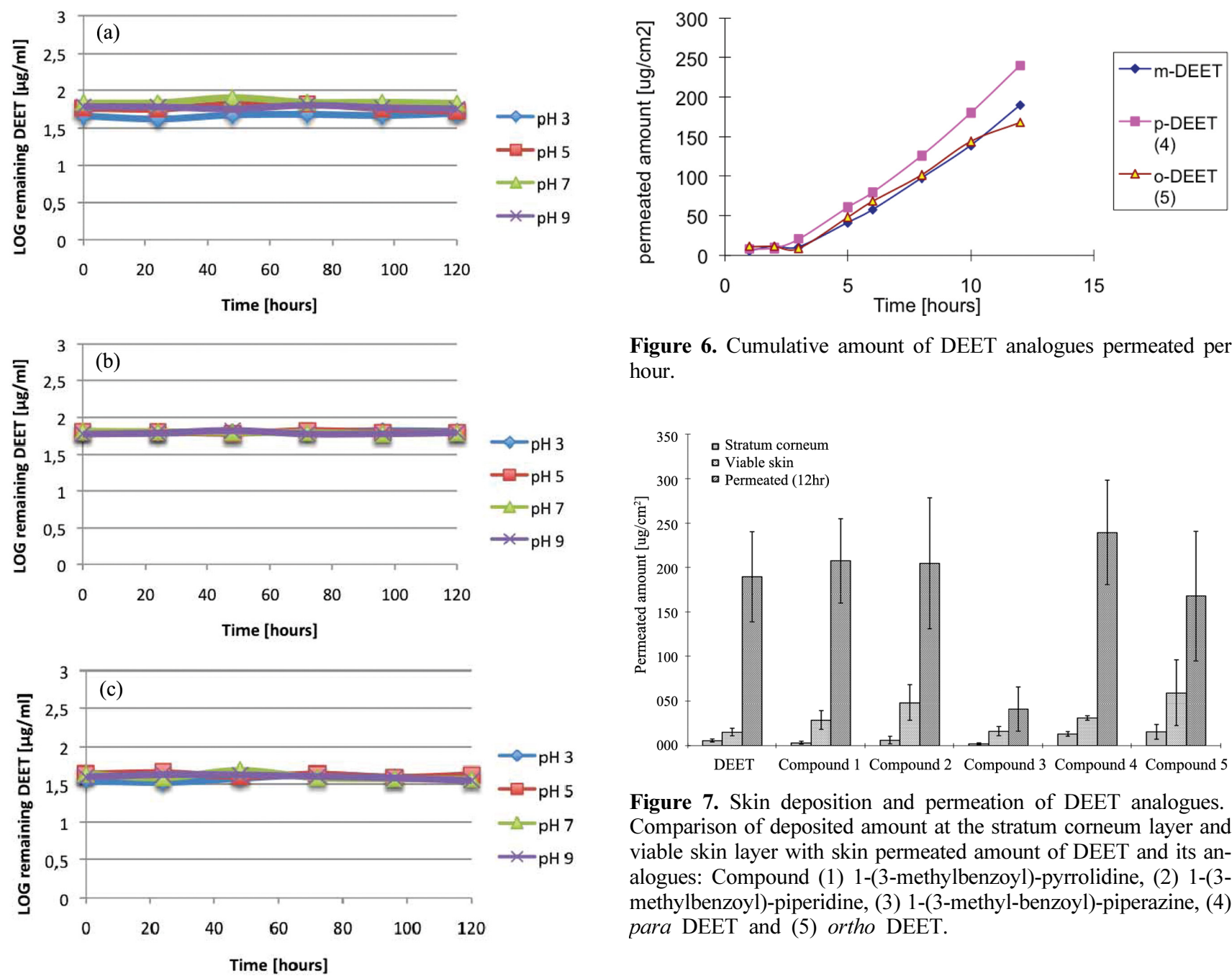

Figure 6. Cumulative amount of DEET analogues permeated per hour.

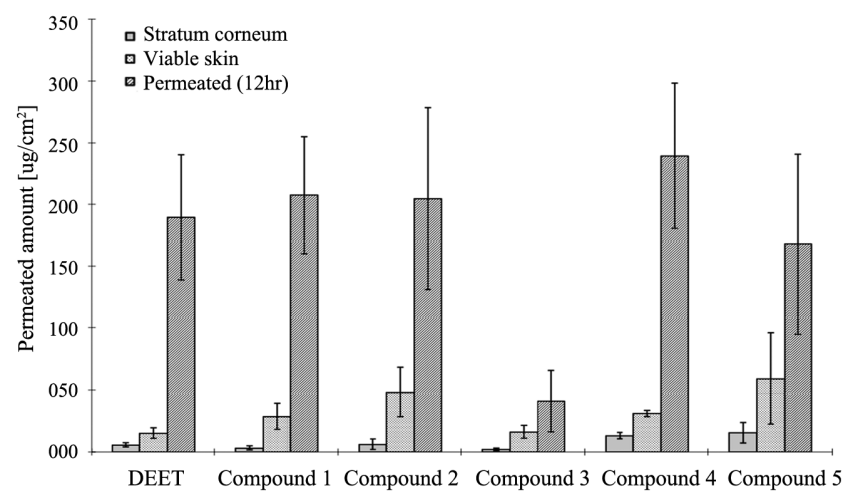

Figure 7. Skin deposition and permeation of DEET analogues. Comparison of deposited amount at the stratum corneum layer and viable skin layer with skin permeated amount of DEET and its analogues: Compound (1) 1-(3-methylbenzoyl)-pyrrolidine, (2) 1-(3methylbenzoyl)-piperidine, (3) 1-(3-methyl-benzoyl)-piperazine, (4) para DEET and (5) ortho DEET.

Figure 5. pH stability of (a) ortho DEET, (b) para DEET and (c) meta DEET at pHs 3, 5, 7 and 9.

(5) which are para-DEET and ortho-DEET respectively, differ in their permeation as well as deposition profile although statistical significance was not shown at the $p<0.05$ level. The

\section{Skin permeation and deposition study}

Figure 6 shows the rat skin permeation profiles of DEET analogues in aqueous solution, using Keshary-Chien permeation cells at $37^{\circ} \mathrm{C}$. After a time lag, the permeation of DEET through the skin followed a zero-order kinetics up to 12 hours. Although statistical significance was not shown at $p<0.05$, the ortho-DEET permeated the least as shown in the permeation rate of $16.54 \mu \mathrm{g} / \mathrm{cm}^{2} / \mathrm{hr}$ in comparison to 22.99 and $28.39 \mu \mathrm{g} /$ $\mathrm{cm}^{2} / \mathrm{hr}$ of the meta and para analogues respectively.

Skin deposition and permeation profiles of the DEET analogues are shown in Figure 7. Compared to meta-DEET, Compound (1), 1-(3-methylbenzoyl)-pyrrolidine and Compound (2) 1-(3-methylbenzoyl)-piperidine exhibited similar permeation profiles as meta-DEET while Compound (3) alias 1-(3-methylbenzoyl)-piperazine permeated the least due to its high hydrophilic character (Jahn et. al., 2010). Compounds (4) and ortho analogue permeated less while the para analogue resembled the meta analogue better. According to a previous study, the complete protection time(CPT) in terms of mosquito repellency of the ortho and para analogues were longer than the meta analogue, indicating that the two were more effective than the commercially used meta analogue (Jahn et. al., 2010). The fact that the ortho DEET permeated the least indicates that the ortho analogue could exhibit less toxicity while maintaining the efficacy of the meta DEET. Moreover, the fact that the ortho analogue exhibited stability similar to the other two analogues makes it a feasible candidate for commercial use.

\section{Conclusion}

The three analogues, ortho, para and meta-DEET were stable at temperatures ranging from $30^{\circ}$ to $70^{\circ} \mathrm{C}$ as well as in the 
$\mathrm{pH}$ ranges of 3 to 9. The fact that ortho-DEET exhibited similar repellency to the commercially used meta analogue while permeating less through skin and existing in a solid state at room temperature, makes it an attractive candidate for a safer and more effective mosquito repellent.

\section{Acknowledgements}

This work has supported by the Basic Research Program of the Korea Science and Engineering Foundation (grant number R01-2006-000-11230-0) and the 2009 Dongseo Frontier Project (S.H.Park).

\section{References}

Barnard, D.R., Xue, R.D., 2004. Laboratory evaluation of mosquito repellents against Aedes albopictus, Cules nigripalpus, and Ochierotatus triseriatus (Diptera:Culicidae). J Med Entomol. 41, 726-730.

Bernier, U.R., Kline, D.L., Barnard, D.R., Schreck, C.E., Yost, R.A., 2000. Analysis of human skin emanations by gas chromatography/mass spectrometry. 2. Identification of volatile compounds that are candidate attractants for the yellow fever mosquito (Aedes Aegypti). Anal Chem. 72(4), 747-756.

Bidlingmayer, W.L., 1994. How mosquitoes see traps: role of visual responses. J Am Mosq Control Assoc. 10(2 Pt 2), 272279.

Corazza, M., Borghi, A., Zampino, M.R., Virgili, A., 2005. Allergic contact dermatitis due to an insect repellent: double sensitization to picaridin and methyl glucose dioleate. Acta Derm Venereol. 85, 264-265.

Ditzen, M., Pellegrino, M., Vosshall, L.B., 2008. Insect odorant receptors are molecular targets of the insect repellent DEET.
Science. 319, 1838-1842.

Dogan, E.B., Ayres, J.W., Rossignol, P.A., 1999. Behavioural mode of action of DEET: inhibition of lactic acid attraction. Med Vet Entomol. 13(1), 97-100.

Gubler, D.J., 2001. Human arbovirus infections worldwide. Ann N Y Acad Sci. 951, 13-24.

Jahn, A., Kim, S.Y., Choi, J.H., Kim, D.D., Ahn, Y.J., Yong, C.S., Kim, J.S., 2010. An improved bioassay for mosquito repellency: Method validation with DEET analogues. J. Pharm. Pharmacol. 62(1), 91-97.

Jensennius, M., Pretorius, A.M., Clarke, F., Myrvang, B., 2005. Repellent efficacy of four commercial DEET lotions against Amblyomma hebraeum (acari: Ixodidae), the principal vector of rickettsia, Africae in southern Africa. Trans R Soc Trop Med Hyg. 99(9), 708-711.

Katritzky, A.R., Wang, Z., Slavov, S., Tsikolia, M., Dobchev, D., Akhmedov, N.G., Hall, C.D., Bernier, U.R., Clark, G.G., Linthicum, K.J., 2008. Synthesis and bioassay of improved mosquito repellents predicted from chemical structure. Proc Natl Acad Sci U S A. 105(21), 7359-7364.

Katz, T.M., Miller J.H., Hebert, A.A., 2008. Insect repellents: Historical perspectives and new developments. Journal of the American Academy of Dermatology. 58(5), 865-871.

Li, S., Picimbon, J.F., Ji, S., Kan, Y., Chuanlian, Q., Zhou, J.J., Pelosi, P., 2008. Multiple functions of an odorant-binding protein in the mosquito aedes aegypti. Biochem BiophysRes Commun. 372(3), 464-468.

Schofield, S., Tepper, M., Gadawski, R., 2007. Laboratory and field evaluation of the impct of exercise on the performance of regular and polymer-based deet repellents. J Med Entomol. 44, 1026-1031.

Syed, Z., Leal, W.S., 2008. Mosquitoes smell and avoid the insect repellent DEET. Proc Natl Acad Sci USA. 105(36), 1359813603. 